\title{
Midterm results of sildenafil therapy in two complex patients with elevated pulmonary artery pressure after cavopulmonary connection
}

\author{
Jacek Białkowski, Małgorzata Szkutnik, Roland Fiszer \\ Congenital Heart Diseases and Pediatric Cardiology Department, Medical University of Silesia, Silesian Center for Heart Diseases, Zabrze, \\ Poland
}

Postep Kardiol Inter 2013; 9, 3 (33): 262-264

DOI: 10.5114/pwki.2013.37506

\begin{abstract}
Two cases are presented. In the first patient (8-year-old boy) after Glenn operation without closure of pulmonary outflow from the common ventricle (SV-PA) despite transcatheter SV-PA closure increased mean pulmonary artery pressure (mPAP) (22 mm Hg) remained. After 6 months of sildenafil therapy he was catheterized again. His mPAP dropped to $10 \mathrm{~mm} \mathrm{Hg}$ and a Fontan operation was subsequently performed. The second patient was a 25 -year-old man, 20 years after a Fontan operation, presenting protein losing enteropathy and severe heart failure. All syndromes diminished significantly after medical therapy with sildenafil. Good clinical results of sildenafil therapy were maintained after 1 and 4 years of treatment. Chronic therapy with sildenafil can be beneficial in patients before and after the Fontan operation with elevated pulmonary artery pressure.
\end{abstract}

Key words: sildenafil, Fontan operation.

\section{Introduction}

Optimal therapy for patients with a functionally single ventricle still remains controversial. Surgical options to improve saturation of these patients are cavopulmonary connections (Fontan type operations). The main hemodynamic condition in such patients should be good ventricular function, low pulmonary pressure and low pulmonary resistance. The aim of this communication is to present midterm results of therapy with a pulmonary vasodilator - sildenafil in two patients with a single right ventricle after the Fontan or hemiFontan operation with elevated pulmonary artery pressure. Recently we discussed this problem in an internet edited issue of the medical journal Congenital Cardiology Today [1] and preliminary results of this therapy were published in Kardiologia Polska [2, 3]. Now mid-term follow-up of these patients is available.

\section{Case reports}

\section{Case 1}

A 7-year-old boy with mitral atresia, single ventricle, and malposition of great arteries was treated from the begin- ning in our center. His body weight was 17.5 kg. In infancy pulmonary artery banding and surgical atrial septectomy (Blalock-Hanlon) was done. A second operation Glenn procedure without closure of pulmonary outflow from common ventricle (SV-PA) was performed. During the subsequent 6 years he was doing relatively well, with good ventricular contractility, without heart failure, with moderate desaturation (81\%). At the age of 7 years he was admitted to our department for cardiac catheterization (qualification for Fontan completion). This examination revealed increased mean pulmonary artery pressure (mPAP) $(22 \mathrm{~mm} \mathrm{Hg})$, good pulmonary artery anatomy, and increased pulmonary artery flow because of opened native single ventricle-pulmonary artery outflow (SV-PA). QP/QS was calculated as 3/1. During the same catheterization pulmonary outflow (previous banding place) was closed with a $5 \mathrm{~mm}$ Amplatzer Atrial Septal Occluder introduced through the right jugular vein. Control hemodynamic assessment revealed no change in pulmonary artery pressure after device outflow closure. After the procedure sildenafil was introduced $(2 \times 12.5 \mathrm{mg} /$ day $)$ to prepare the pulmonary vascular bed for the Fontan operation (elevated mPAP pressure was a risk factor). He was

\section{Corresponding author:}

Prof. Jacek Białkowski MD, PhD, Congenital Heart Diseases and Pediatric Cardiology Department, Medical University of Silesia, Silesian Center for Heart Diseases, 2 Szpitalna St, 41800 Zabrze, Poland, tel.: +48 3227134 01, e-mail: jabi_med@poczta.onet.pl Received: 15.04.2013, accepted: 12.06.2013. 
also treated with captopril, spironolacton and carvedilol. After 6 months he was catheterized again. His pulmonary pressure dropped more than half (mPAP $10 \mathrm{~mm} \mathrm{Hg}$ ), QP/QS was 0.43 , PVR 2 Wood units. After 3 months he underwent a total cavopulmonary connection (TCPC) operation performed with an extracardiac tunnel $(18 \mathrm{~mm})$. The postoperative period was complicated with low cardiac output syndrome and right pleurothorax which needed 2 pleurocenteses. During the next days his clinical condition improved and he was discharged home 17 days after the operation on sildenafil $(2 \times 12.5 \mathrm{mg})$ and standard medication (captopril, furosemide, spironolacton, acenocoumarol). He remains in good condition after 1 year of follow-up [3].

\section{Case 2}

A 21-year-old male patient from another center was admitted to our department because of severe heart failure and protein losing enteropathy (PLE) as the consequence of failing Fontan. The initial diagnosis was mitral atresia, single ventricle and malposition of the great arteries. In infancy pulmonary artery banding and surgical atrial septectomy (Blalock-Hanlon) was performed. The second operation was performed at the age of 4 years (hemi-Fontan) and 1 year later, completion of Fontan - TCPC, without fenestration. During later follow-up he developed PLE with ascites. At the age of 15 years he was catheterized and mean pulmonary artery pressure (mPAP) was $27 \mathrm{~mm} \mathrm{Hg}$. Pulmonary artery anatomy was good. During the next 5 years his condition deteriorated - increasing peripheral swellings, ascites and cachexia. Because of ascites abdominal hernia developed which was closed surgically 1 month before his first admission to our department. At that moment there was still severe ascites with spontaneous drainage of peritoneal liquid from the postsurgical scar of the previously closed hernia. The circumference of the abdomen at that time was $130 \mathrm{~cm}$ with body weight $65 \mathrm{~kg}$. He also had a visible dilated jugular vein, hepatomegaly $(6 \mathrm{~cm})$ and peripheral edema, desaturation (85\%) and pleurothorax of the right lung. He was in NYHA class III. After examinations (ECHO, TC, MRI) good single ventricle function (EF 55\%) and pulmonary anatomy was confirmed. During pleurocentesis $1400 \mathrm{ml}$ of liquid was removed. Until that time he was treated with furosemide, hydrochlorothiazide, spironolacton, carvedilol and inhibace. Because of no clinical improvement we decided to introduce sildenafil $3 \times 25 \mathrm{mg}$ daily. After 4 weeks of such therapy we observed spectacular clinical improvement (NYHA class II). He lost $5 \mathrm{~kg}$ of fluids, circumference of the abdomen was smaller $(85 \mathrm{~cm})$, the postsurgical wound closed successfully, and the liver diminished $(3 \mathrm{~cm})$. He was discharged home on this therapy. After 3 months he lost another $14 \mathrm{~kg}$ of fluid and his abdomen progressed to be smaller. Oxygen consumption test improved from $14 \mathrm{ml} / \mathrm{kg} / \mathrm{min}$ initially to $28 \mathrm{ml} / \mathrm{kg} / \mathrm{min} 1$ year after sildenafil therapy. This clinical improvement persisted after 4 years of follow-up with only mild ascites and pleurotho- rax. Now he can continue his university studies. He refused control cardiac catheterization for assessment of pulmonary pressure [2].

\section{Discussion}

The administration of pulmonary vasodilators such as sildenafil has been shown to reduce elevated pulmonary artery pressure [4]. There is increasing evidence that at least half of post-Fontan patients have increased pulmonary vascular resistance as a consequence of pulmonary endothelial dysfunction. Goldberg et al. [5] suggested that maneuvers which increase cardiac output and lower central venous pressure can improve Fontan circulation. In case of PLE, with serum albumin level $<2.0 \mathrm{~g} / \mathrm{dl}$, he recommended treatment with sildenafil, CD-budesonite or Fontan surgical revision or heart transplantation. Moreover, the same author in a recently published paper showed that sildenafil may be a useful therapy to improve or maintain ventricular performance in selected patients after the Fontan operation [6]. Our observations in patients presented here as well as that of Deal and Jacobs [7] suggested that chronic pulmonary vasodilator therapy, in addition to chronic diuretics, may become part of routine long-term therapy in selected Fontan patients. What we noticed in our patients was not better ventricular performance in relation to sildenafil therapy but reduced pulmonary artery resistance resulting in clinical improvement. Meadows and Jenkins [8] in their comprehensive review summarized experience with evaluation, management and treatment of PLE in 18 patients from Boston Children Hospital (without application of sildenafil). Our cases and experience of others [9] indicate that sildenafil can be used safely and effectively in the treatment of patients with failing Fontan circulations. Interestingly, Ovaert et al. [10] in her study failed to show significant improvement after 3 months of treatment with bosentan (another type of pulmonary vasodilator) in 10 patients with failing Fontan.

In summary, patients with a single ventricle and elevated pulmonary artery pressure, both before and after the Fontan operation (failing Fontan), can benefit in our opinion from chronic sildenafil treatment.

\section{References}

1. Bialkowski J, Szkutnik M. Successful chronic treatment with sildenafil in two patients with functionally single ventricle after Fontan/hemi-Fontan procedures. CCT 2013; April, 12-13.

2. Bialkowski J, Rycaj J, Glowacki J, et al. Successful chronic treatment with sildenafil in a patient with end-stage failure following Fontan procedure. Kardiol Pol 2011; 69: 302-304.

3. Salas Llamas JP, Szkutnik M, Fiszer R, Bialkowski J. Treatment of elevated pulmonary artery pressure in a child after Glenn procedure: transcatheter closure of pulmonary artery banding with subsequent sildenafil therapy. Kardiol Pol 2012; 70: 201-203.

4. Lu X, Xiong C, Shan G, et al. Impact of sildenafil therapy on pulmonary arterial hypertension in adults with congenital heart disease. Cardiovasc Ther 2010; 28: 350-355. 
5. Goldberg DJ, Dodds K, Rychlik J. Rare problems associated with the Fontan circulation. Cardiol Young 2010; 20 (suppl 3): 113-119.

6. Goldberg DJ, French B, Szwast A, et al. Impact of sildenafil on echocardiographic indices of myocardial performance after the Fontan operation. PediatrCardiol 2012; 33; 689-696.

7. Deal B, Jacobs M. Management of the failing Fontan circulation. Heart 2012; 98: 1098-1104.

8. Meadows J, Jenkins K. Protein-losing entheropathy: integrating a new disease paradigm into recommendations for prevention and treatment. Cardiol Young 2011; 21; 363-377.

9. Reinhardt Z, Uzun O, Bhole V, et al. Sildenafil in the management of the failing Fontan circulation. Cardiol Young 2010; 20: 522-525.

10. Ovaert C, Thijs D, Dewolf D, et al. The effect of bosentan in patients with failing Fontan circulation. Cardiol Young 2009; 19: 331-339. 